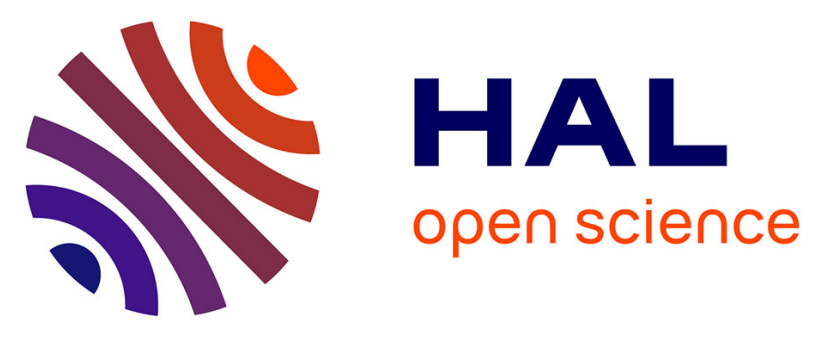

\title{
Suppression of Parasitic Nonlinear Processes in Spontaneous Four-Wave Mixing with Linearly Uncoupled Resonators
}

Federico Andrea Sabattoli, Federico Andrea Sabattoli, Houssein El Dirani, Laurène Youssef, Francesco Garrisi, Davide Grassani, Luca Zatti, Camille Petit-Etienne, Erwine Pargon, J. E. Sipe, et al.

\section{To cite this version:}

Federico Andrea Sabattoli, Federico Andrea Sabattoli, Houssein El Dirani, Laurène Youssef, Francesco Garrisi, et al.. Suppression of Parasitic Nonlinear Processes in Spontaneous Four-Wave Mixing with Linearly Uncoupled Resonators. Physical Review Letters, 2021, 127 (3), 10.1103/PhysRevLett.127.033901 . hal-03323869

\section{HAL Id: hal-03323869 https://hal.science/hal-03323869}

Submitted on 23 Aug 2021

HAL is a multi-disciplinary open access archive for the deposit and dissemination of scientific research documents, whether they are published or not. The documents may come from teaching and research institutions in France or abroad, or from public or private research centers.
L'archive ouverte pluridisciplinaire HAL, est destinée au dépôt et à la diffusion de documents scientifiques de niveau recherche, publiés ou non, émanant des établissements d'enseignement et de recherche français ou étrangers, des laboratoires publics ou privés. 


\title{
Suppression of Parasitic Nonlinear Processes in Spontaneous Four-Wave Mixing
}

\author{
Federico Andrea Sabattoli, ${ }^{1, *}$ Houssein El Dirani, $,{ }^{2}, \dagger$ Laurène Youssef, ${ }^{3}$ Francesco \\ Garrisi, ${ }^{1}$ Davide Grassani, ${ }^{1}$ Luca Zatti, ${ }^{1}$ Camille Petit-Etienne, ${ }^{3}$ Erwine Pargon, ${ }^{3}$ J.E. \\ Sipe, ${ }^{4}$ Marco Liscidini, ${ }^{1}$ Corrado Sciancalepore,,${ }^{2} \ddagger$ Daniele Bajoni, ${ }^{5}$ and Matteo Galli ${ }^{1}$ \\ ${ }^{1}$ Dipartimento di Fisica, Università degli Studi di Pavia, Via Bassi 6, 27100 Pavia, Italy \\ ${ }^{2}$ Université Grenoble Alpes, CEA-LETI, 38054 Grenoble, France \\ ${ }^{3}$ Université Grenoble Alpes, CNRS, LTM, 38000 Grenoble, France \\ ${ }^{4}$ Department of Physics, University of Toronto, 60 St. George Street, Toronto, Ontario M5S 1A7, Canada \\ ${ }^{5}$ Dipartimento di Ingegneria Industriale e dell'Informazione, \\ Università degli Studi di Pavia, via Ferrata 1, 27100 Pavia, Italy
}

(Dated: November 14, 2020)

\begin{abstract}
We report an enhancement of over $10^{4}$ in the signal-to-noise ratio characterizing the generation of identical photon pairs in a ring resonator system. Parasitic noise, associated with single pump spontaneous four-wave mixing, is essentially eliminated by employing a novel system design involving two resonators that are linearly uncoupled but nonlinearly coupled. This opens the way to a new class of integrated devices exploiting the unique properties of identical photon pairs in the same optical mode.
\end{abstract}

Nonlinear optics has been widely exploited to produce nonclassical states of the electromagnetic field. There has been a recent focus on the use of CMOS compatible materials, such as silicon and silicon nitride, which are characterized by a strong third-order optical response that can be further enhanced by the light confinement attainable in integrated microresonators [1]. With the fabrication quality and technological maturity achieved in the last decade, integrated devices based on these materials offer the promise of efficient generation of entangled photons $[2-6]$, heralded single-photons $[7,8]$ and squeezed light $[9,10]$.

A key third-order nonlinear process is dual-pump spontaneous four-wave mixing (d-SFWM), in which pump lasers at $\omega_{1}$ and $\omega_{2}$ are used to generate photons at $\omega_{T}=\left(\omega_{1}+\omega_{2}\right) / 2$. This process can be used to generate single-mode squeezed light, a central resource in the development of continuous-variable quantum computing [11]. While conventional micrometric ring resonators have been used to enhance d-SFWM [12], their multimode nature inevitably also leads to the amplification of unwanted nonlinear phenomena, such as singlepump spontaneous four-wave mixing (s-SFWM). This is a problem: as shown in Fig. 1(a), if the two pumps are resonant with the microring, the generation of photon pairs within a target resonance $\left(\omega_{T}\right)$ through d-SFWM occurs along with two s-SFWM processes. Both of these parasitic s-SFWM processes lead to the generation of two photons, with one, and only one, of them in the mode at $\omega_{T}$. This acts as a source of noise, leading to a degradation of the squeezed light [10]).

\footnotetext{
* federicoandrea.sabattoli01@ateneopv.it

$\dagger$ Now at STMicroelectronics, 38926 Crolles Cedex, France

$\ddagger$ Now at SOITEC SA, Parc technologique des Fontaines, Chemin des Franques, 38190 Bernin, France
}

To quantitatively characterize these nonlinear parasitic processes, in Fig.1(b) we plot the photon generation rates (measured as described in the Supplemental Material) due to the d-SFWM and s-SFWM processes in an integrated silicon microring resonator (length $372 \mu \mathrm{m}$ and quality factor around $6 \cdot 10^{4}$ ). As expected, the generation rate for the d-SFWM is unchanged as long as the product of the two pump powers is held fixed, while the generation rate of the s-SFWM processes scale quadratically with the appropriate pump powers [13]. The generation rates of the parasitic processes, given by the sum of the two s-SFWM rates, can even be one order of magnitude larger than the generation rate of the d-SFWM process, with a maximum signal-to-noise ratio (SNR) of only about 2 when the two pump powers are equal.

Strategies have been proposed to overcome this intrinsic limit. In one approach, the two pump lasers are slightly detuned from resonance, such that the s-SFWM rates are suppressed [10], but with a trade-off between SNR and generation rate. Alternatively, one can use systems composed of two or more linearly coupled resonators to engineer the spectral position of the resonant modes, with nonlinear phenomena selectively enhanced or suppressed $[9,14,15]$. While this can be very effective, the suppression of the parasitic processes is limited by the achievable resonant splitting of the coupled modes.

In 2019, Menotti et al. proposed a new strategy [16], in which two optical resonators are coupled solely through the nonlinear interaction. The system is characterized by two independent sets of resonances that can be tuned to selectively enhance or suppress different nonlinear phenomena. In Fig. 2(b) we represent a sketch of the realisation of d-SFWM in such a system. Two laser pumps are tuned to resonances of the first resonator, leading to the generation of photon-pairs within a target resonance of the second resonator. Yet s-SFWMs are expected to be suppressed, for the overall field enhancement 
(a)

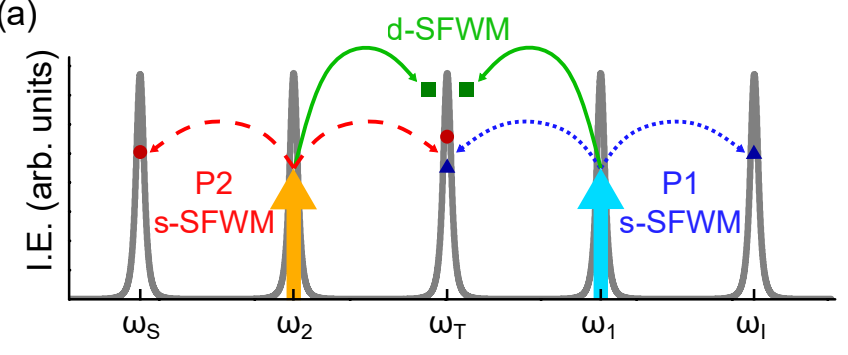

(b)

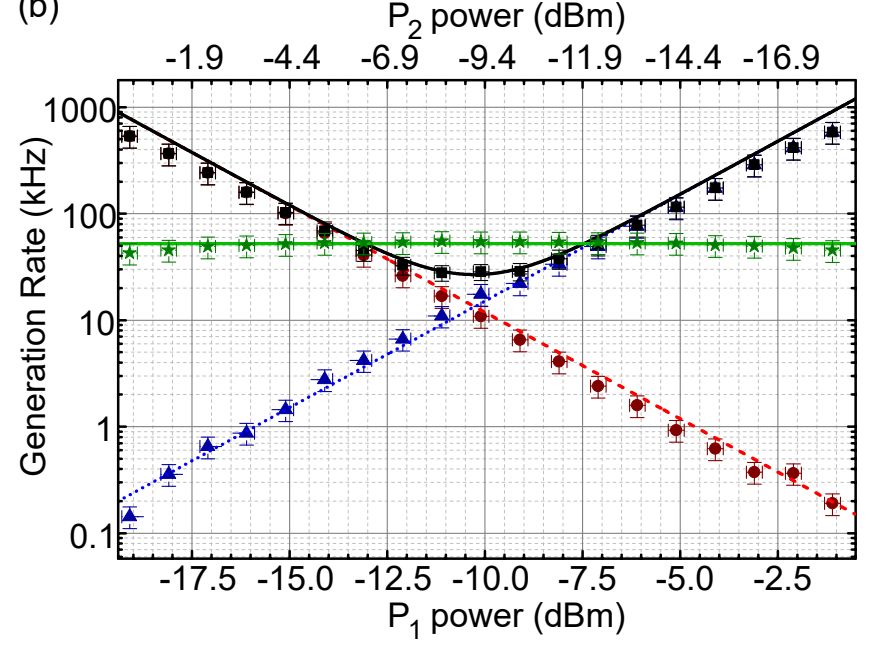

FIG. 1. (a) Sketch of the s-SFWM and d-SFWM processes occurring in a single microring resonator when two laser pumps $\mathrm{P}_{1}$ and $\mathrm{P}_{2}$ are aligned to two resonances $\left(\omega_{1}\right.$ and $\left.\omega_{2}\right)$. All the processes are resonant and exploit a significant intensity enhancement (I.E.). (b) Measurement of the pair generation rate in a single silicon microring resonator when the product of the powers of the two pumps is fixed. The blue triangles and the red circles show the s-SFWM processes pumped by the lasers at $\omega_{1}$ and $\omega_{2}$, respectively. The black squares show the sum of the parasitic process rates, and the green stars show the d-SFWM generation rate. The measures are compared with the theoretical calculation (shown with the dotted blue line for the $\mathrm{P}_{1}$-pumped s-SFWM, the dashed red line for the $\mathrm{P}_{2}$-pumped s-SFWM, and the green line for d-SFWM.) See Supplemental Material for the experimental details, and Menotti et al. [16] for the theoretical calculations.

at $\omega_{S}=2 \omega_{2}-\omega_{T}$ and $\omega_{I}=2 \omega_{1}-\omega_{T}$ is strongly reduced by the absence of three resonances satisfying energy conservation. In this letter we experimentally demonstrate this scheme.

The device is presented in Fig.2(a): two silicon-oninsulator (SOI) racetrack resonators (waveguide crosssection $600 \times 220 \mathrm{~nm}^{2}$ ) with different lengths $\mathcal{L}_{1}=372 \mu \mathrm{m}$ and $\mathcal{L}_{2}=366 \mu \mathrm{m}$, are located side-by-side forming a directional coupler (DC) of length $L_{D C}=92 \mu \mathrm{m}$, chosen to minimise cross-transmission and achieve isolation of the two resonators. Thus one set of modes is associated with Resonator $1\left(\mathrm{R}_{1}\right)$, and another with Resonator $2\left(\mathrm{R}_{2}\right)$. During the fabrication process, a $\mathrm{H}_{2}$ thermal annealing was used to smooth the sidewalls of silicon waveguides
[17]: this leads to the reduction of the propagation losses and, thus, to the realisation of resonators with high quality factors [18]. The optical characterization is shown in Fig. 2(c). The resulting two independent sets of resonances are clearly observed by injecting light from a tunable laser in the ports $T_{1}$ or $T_{2}$ and detecting the optical response at the ports $\mathrm{T}_{6}$ and $\mathrm{T}_{3}$, respectively. The free spectral range (FSR) at $1550 \mathrm{~nm}$ is around $1.566 \mathrm{~nm}$ and $1.595 \mathrm{~nm}$ for $\mathrm{R}_{1}$ and $\mathrm{R}_{2}$ respectively. The loaded quality factors are around $6 \cdot 10^{4}$ and $3 \cdot 10^{5}$, with the difference due to the different number of waveguides coupled to the racetracks (two to $R_{1}$, and one to $R_{2}$ ). The optical isolation is at least $20 \mathrm{~dB}$ over a $40-\mathrm{nm}$ bandwidth, as observed from the transmission spectrum $\mathrm{T}_{1} \rightarrow \mathrm{T}_{3}$.

(a)

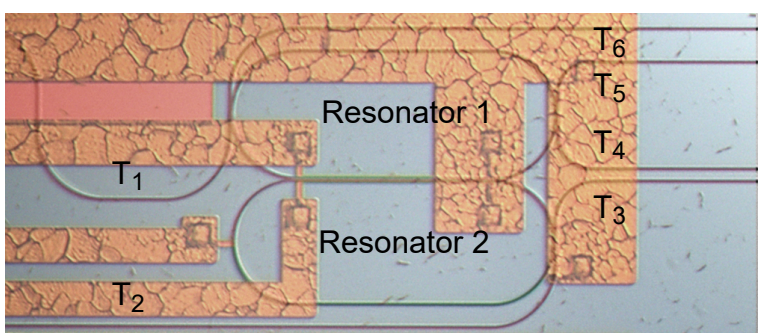

(b)
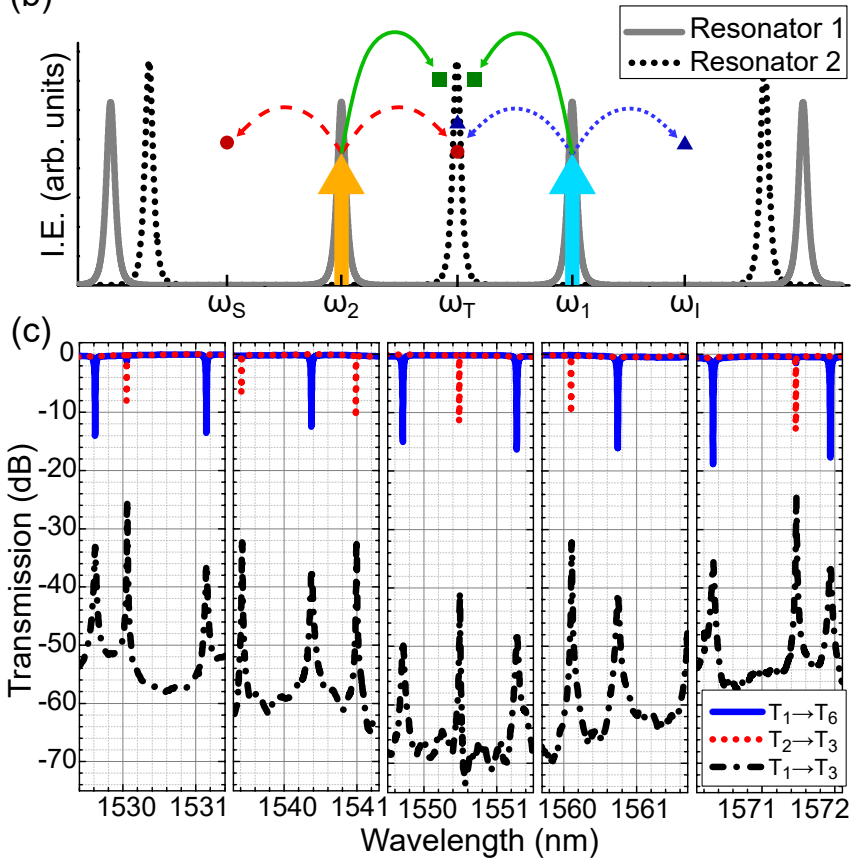

FIG. 2. (a) Optical image of the device with two linearly uncoupled racetracks. A second image of the device without the electrical circuits has been superimposed to show the position of waveguides, which are covered by the metal. (b) Sketch of the s-SFWM and d-SFWM processes occurring in the device when two laser pumps are aligned to two resonances $\left(\omega_{1}\right.$ and $\left.\omega_{2}\right)$. Single-pump processes which generate photons at $\omega_{T}$ are suppressed due to the weak field enhancement experienced at the spectral position where energy conservation is preserved. (c) Linear characterization of the device. 


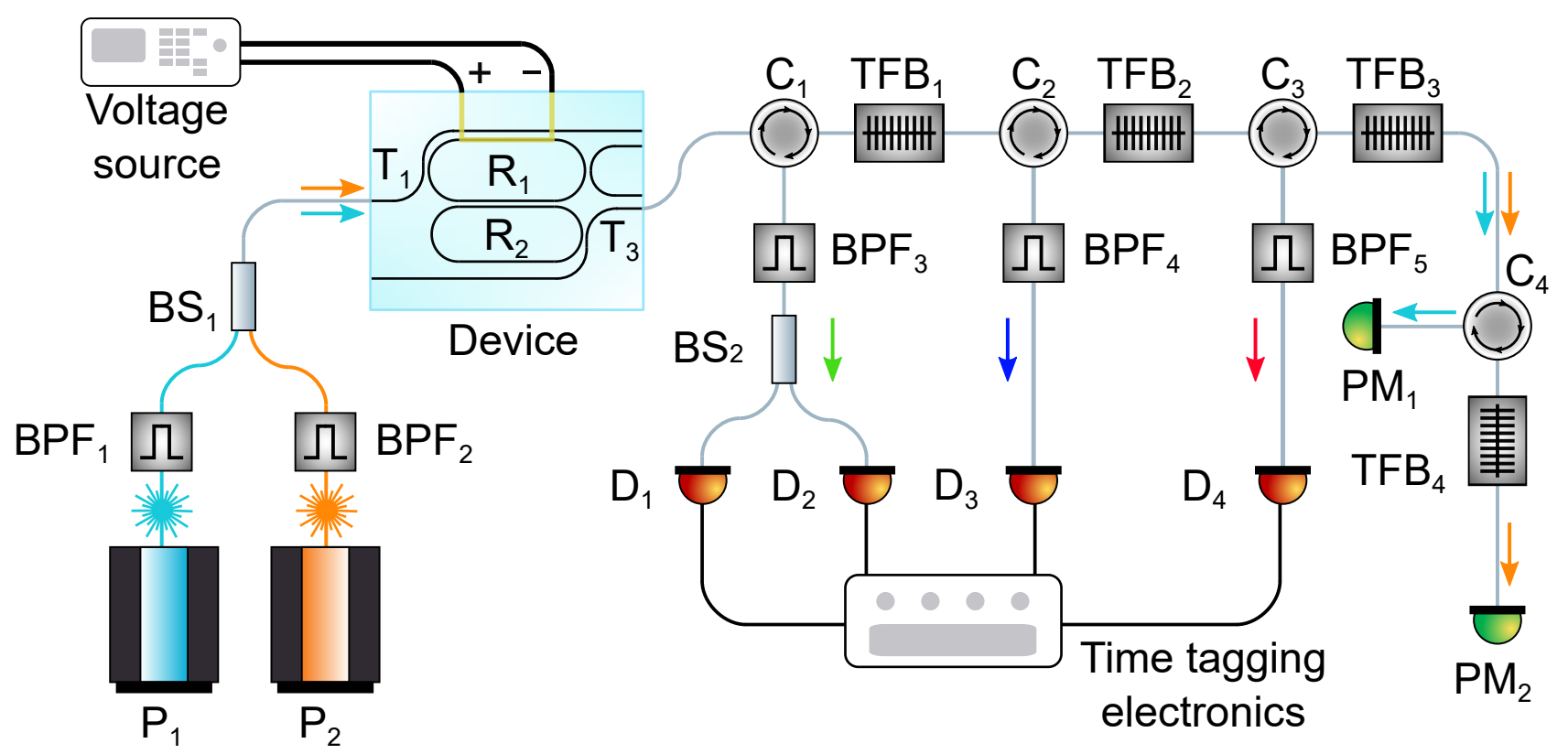

FIG. 3. Experimental set-up used to measure the coincidence rates. Pump light from two lasers, combined into the same fiber through a beam splitter $\mathrm{BS}_{1}$, is coupled to resonator $\mathrm{R}_{1}$, the resonances of which are tuned by changing the voltage applied to the heater. Photons generated through d-SFMW and s-SFWM are collected with a tapered fiber, and sorted by frequency using tunable fiber Bragg filters $\left(\mathrm{TFB}_{1}-\mathrm{TFB}_{3}\right)$ and circulators $\left(\mathrm{C}_{1}-\mathrm{C}_{3}\right)$. They are then detected using four superconductive single photon detectors $\left(\mathrm{D}_{1}-\mathrm{D}_{4}\right)$. The correlations between the detection times are recorded and analysed through time tagging electronics. The spectral alignment of the lasers with the device is checked with two power meters $\left(\mathrm{PM}_{1}\right.$ and $\left.\mathrm{PM}_{2}\right)$, with an additional fiber Bragg filter $\left(\mathrm{TFB}_{4}\right)$ and circulator $\left(\mathrm{C}_{4}\right)$ used to separate the two beams. Bandpass filters $\mathrm{BPF}_{1}$ and $\mathrm{BPF}_{2}$ are used to eliminate amplified spontaneous emission from the output of the pump lasers, and $\mathrm{BPF}_{3}-\mathrm{BPF}_{5}$ to filter out residual pump photons.

The two sets of resonances can be controlled independently by means of two electric heaters fabricated with a TiN layer on top of the silica cladding, and the electrical contact is realised with $\mathrm{AlCu}$ electric wires deposited on top of the chip.

Measurements of the nonlinear processes are realised with the experimental set-up presented in Fig.3. The light from two tunable lasers (Santec TSL710 and TSL510) is coupled using a collimator and lens to match the mode field diameter of the edge coupling tip on the chip, after two band-pass filters $\mathrm{BPF}_{1}$ and $\mathrm{BPF}_{2}$ (FWHM around $8 \mathrm{~nm}$ ) have been used to filter out the amplified spontaneous emission from the lasers. The spectral position of the resonances of $R_{1}$ is controlled by using an external voltage source (Keithley 2400 Source Meter) and a multi-contact wedge. The generated photon pairs are collected by a tapered fiber coupled to the port $\mathrm{T}_{3}$ of the device, and the fiber is connected to the circulator $\mathrm{C}_{1}$. A tunable fiber Bragg filter $\left(\mathrm{TFB}_{1}\right)$ is used in reflection [65 pm 3dB-bandwidth (BW) and $40 \mathrm{~dB}$ of extinction ratio (ER)] to route photons at frequencies around $\omega_{T}$ towards the detection system. Another band-pass filter $\left(\mathrm{BPF}_{3}\right)$ with more than $60 \mathrm{~dB}$ of ER filters out any residuals from the pump, and a fiber 50:50 beam splitter splits the photon flux towards the superconductive single-photon detectors $\mathrm{D}_{1}$ and $\mathrm{D}_{2}$ supplied by Photon Spot.
Photons at $\omega_{T}$ can be generated through s-SFWM only if another photon is generated to guarantee the conservation of energy. In particular, s-SFWM pumped by the laser at $\omega_{1}\left(\mathrm{P}_{1}\right.$-SFMW $)$ can generate a photon pair with one photon at $\omega_{T}$ and one at $\omega_{I}$, such that $\omega_{I}=2 \omega_{1}-\omega_{T}$. Similarly, s-SFWM associated with the laser at $\omega_{2}\left(\mathrm{P}_{2^{-}}\right.$ SFMW) can generate one photon at $\omega_{T}$ and one photon at $\omega_{S}$, where $\omega_{S}=2 \omega_{2}-\omega_{T}$. To detect these photons, we use two tunable fiber Bragg filters $\left(\mathrm{TFB}_{2}\right.$ and $\left.\mathrm{TFB}_{3}\right)$ and two band-pass filters $\left(\mathrm{BPF}_{4}\right.$ and $\left.\mathrm{BPF}_{5}\right)$ to route photons at $\omega_{I}$ and $\omega_{S}$ towards superconductive single-photon detectors $\mathrm{D}_{3}$ and $\mathrm{D}_{4}$, respectively. Finally, the alignment of the two laser pumps is checked during the whole experiment using two power meters $\left(\mathrm{PM}_{1}\right.$ and $\left.\mathrm{PM}_{2}\right)$, a circulator $\left(\mathrm{C}_{4}\right)$, and an additional band-pass filter $\left(\mathrm{TFB}_{4}\right)$.

The total insertion losses from the lasers to the waveguide coupled with $R_{1}$ at the port $T_{1}$ are around $-7.8 \pm 0.1$ $\mathrm{dB}$ for the light from both $\mathrm{P}_{1}$ and $\mathrm{P}_{2}$. The losses from the waveguide coupled with $R_{1}$ at the port $T_{3}$ to $D_{1}$ and $\mathrm{D}_{2}$ are around $-10.7 \pm 0.3 \mathrm{~dB}$ and $-10.1 \pm 0.3 \mathrm{~dB}$, respectively, for the photons at $\omega_{T}$; the losses from $\mathrm{T}_{3}$ to $\mathrm{D}_{3}$ are $-11.9 \pm 0.3 \mathrm{~dB}$ for photons at $\omega_{I}$, and $-7.6 \pm 0.3$ $\mathrm{dB}$ from $\mathrm{T}_{3}$ to $\mathrm{D}_{4}$ for photons at $\omega_{S}$. The efficiency of the detectors is $-1.00 \pm 0.05 \mathrm{~dB}$.

We begin by verifying the capability of our structure to suppress s-SFWM. To do that, we measure the vari- 
ation of the efficiency of an s-SFWM process by tuning the two sets of resonances, which leads to a modification of the nonlinear coupling of the modes. We start by adjusting the voltage applied to the heater of $R_{1}$ so that the frequency $\omega_{2}$ of a resonance (vacuum wavelength $\lambda_{2}=1560.916 \mathrm{~nm}$ ) is in the middle of the frequencies of two resonances of $\mathrm{R}_{2}$ (at vacuum wavelengths $\lambda_{T}=1550.495 \mathrm{~nm}$ and $\left.\lambda_{U}=1571.479 \mathrm{~nm}\right)$. This configuration should guarantee a maximum efficiency of $\mathrm{P}_{2^{-}}$ SFWM, since $\omega_{U}=\omega_{S}=2 \omega_{2}-\omega_{T}$. Then, with laser $\mathrm{P}_{1}$ turned off, we change the driving voltage of the heater of $\mathrm{R}_{1}$ to shift its resonances, and we tune $\mathrm{P}_{2}$ at each step to be on resonance with $R_{1}$ at each $\lambda_{2}$ by checking the transmission on the power meter $\mathrm{PM}_{2}$. The tunable fiber Bragg filter $\mathrm{TFB}_{1}$ is aligned to the resonance at $\omega_{T}$ at the beginning of the experiment, since thermal cross-talk leads to a tuning of the resonances of $R_{2}$ within the bandwidth of the filter. On the other hand, the filter $\mathrm{TFB}_{2}$ is tuned to collect the photons generated at $\omega_{S}=2 \omega_{2}-\omega_{T}$, which requires the adjustment of its spectral alignment during the whole experiment. In Fig. 4 we show the attenuation of the single-pump SFWM process with respect to the detuning of the resonances involved, which is estimated from the driving voltage and the characterization of the heater (see Supplemental Material for details). In particular, that detuning is defined as $\Delta \omega_{2}-\left(\Delta \omega_{T}-\Delta \omega_{U}\right) / 2$. We find that the simulation and the data are in good agreement, and exhibit the expected behaviour.

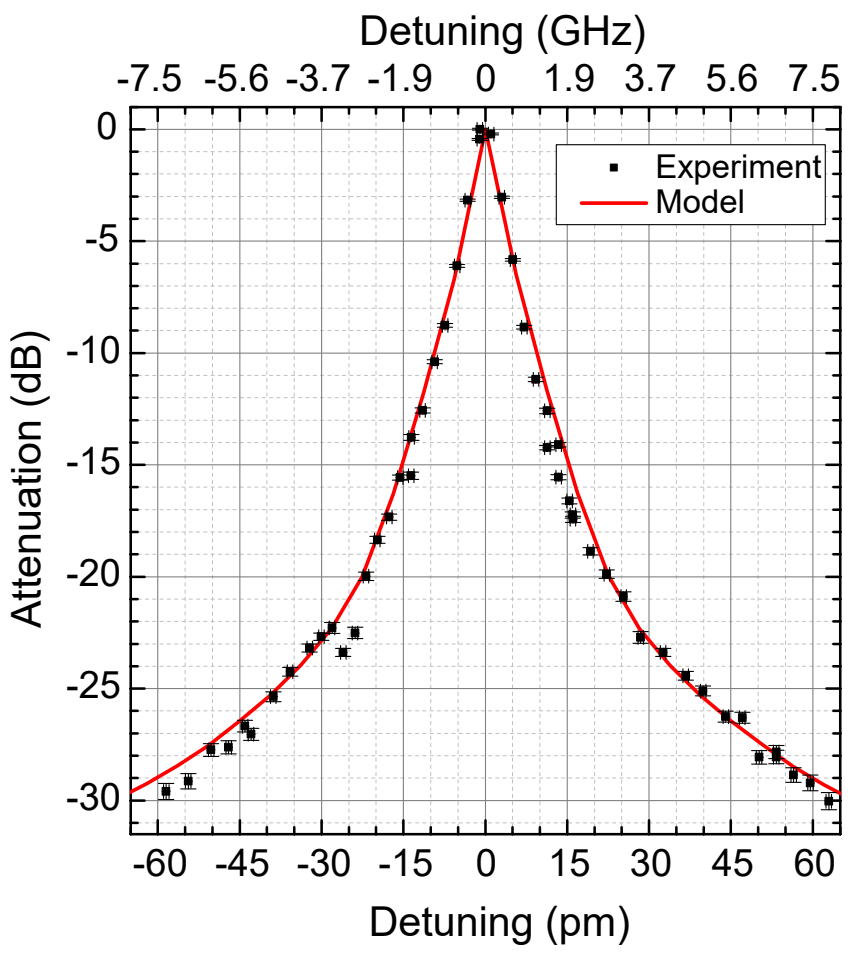

FIG. 4. Plot showing the attenuation of s-SFWM as a function of the detuning of the resonances of the two resonators.
This result shows that by tuning the two sets of resonances one can manipulate the nonlinear coupling of the modes of the resonators, and, consequently, the generation of pairs due to the nonlinear processes occurring in the device. In particular, a detuning of around $65 \mathrm{pm}$ (8.1 $\mathrm{GHz}$, around 13 times the linewidth of the resonances of $\mathrm{R}_{2}$ ) leads to a suppression of around three orders of magnitude with respect to perfect nonlinear coupling.

With the possibility of attenuating the parasitic processes demonstrated, we proceed to the dual-pump SFWM experiment. The laser pumps are aligned to two resonances of $R_{1}$ (vacuum wavelengths $\lambda_{1}=1540.374$ $\mathrm{nm}$ and $\left.\lambda_{2}=1560.742 \mathrm{~nm}\right)$, which are equally spaced in frequency about a resonance of $\mathrm{R}_{2}$ at $\lambda_{T}=1550.491$ nm. This configuration is associated with a detuning of the s-SFWM resonances of around $-160 \mathrm{pm}(-20 \mathrm{GHz})$, and the estimation of the resulting attenuation of the sSFWM processes is around $-37.3 \mathrm{~dB}$. The quality factors of the resonances of $R_{1}$ are around $\mathrm{Q}_{1}=6.7 \cdot 10^{4}$ and $\mathrm{Q}_{2}=5.7 \cdot 10^{4}$, while the resonance of $\mathrm{R}_{2}$ at $\lambda_{T}$ has a much higher quality factor, $\mathrm{Q}_{T}=3.2 \cdot 10^{5}$. We align the tunable filter $\mathrm{TFB}_{1}$ to $\lambda_{T}$, while $\mathrm{TFB}_{2}$ and $\mathrm{TFB}_{3}$ are tuned to route photons at $\lambda_{I}=1530.388 \mathrm{~nm}$ and $\lambda_{S}=1571.129 \mathrm{~nm}$ to $\mathrm{D}_{3}$ and $\mathrm{D}_{4}$, respectively. The analysis of the coincidences on the detectors allows for the recognition of the pairs generated through the different processes. In particular, the d-SFMW should give coincidence between the detection events at $\mathrm{D}_{1}$ and $\mathrm{D}_{2}$. Similarly, the single-pump processes should result in coincident detection: $\mathrm{P}_{1}$-SFWM between the events at $\mathrm{D}_{3}$ and $\mathrm{D}_{1}$ (or $\mathrm{D}_{3}$ and $\mathrm{D}_{2}$ ), and $\mathrm{P}_{2}$-SFWM between the events at $\mathrm{D}_{4}$ and $\mathrm{D}_{1}$ (or $\mathrm{D}_{4}$ and $\mathrm{D}_{2}$ ).

As discussed earlier [16], the efficiency of the d-SFWM process in our device is reduced from what could be achieved with a simple microring, since here the nonlinear interaction occurs only in the directional coupler region shared by the two rings. This decreases both the overlap integral of the fields $(1 / 4)$ and the interaction length $\left(L_{D C} \approx \mathcal{L} / 4\right)$. This would be a limitation in the framework of squeezed-light sources, where high generation efficiency is required, but it could be overcome by working with ultra low-loss waveguides with high modal confinement in silicon nitride $[19,20]$. In such a device one could also increase the pump power, since the twophoton absorption that affects silicon waveguides would not be present [21].

In Fig. 5 we show the result of a coincidence experiment with the current device, where $0.95 \pm 0.05 \mathrm{~mW}$ of optical power is coupled into the waveguide from each pump laser. The data are acquired for 20 minutes, and the his-

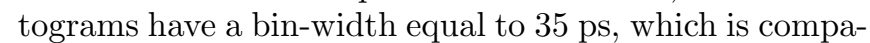
rable with the time jitter of the detectors. The red peak shows that photons arriving on $\mathrm{D}_{1}$ and $\mathrm{D}_{2}$ are emitted at the same time, since their arrival time is correlated: this clearly demonstrates they are emitted through d-SFWM, since any other process that can generate photons at $\lambda_{T}$ cannot be characterized by this temporal correlation. We measure a coincidence rate equal to $164.2 \pm 0.4 \mathrm{~Hz}$, which 
corresponds to an internal generation rate in the second resonator equal to $62 \pm 6 \mathrm{kHz}$, and the CAR is $1190 \pm 10$. The coincidence rate is estimated by integrating the peak within the whole window (and subtracting the noise), while the CAR is measured by taking into account the FWHM of the peak (16 bins of the histogram).

From the histograms in Fig.5 it is also possible to evaluate the photon pairs generated through s-SFWM, which lead to coincidence events between $\mathrm{D}_{1}$ and $\mathrm{D}_{2}$ with $\mathrm{D}_{3}$ or $\mathrm{D}_{4}$. As can be seen, no peaks are clearly visible. In fact, the dark counts, the noise from the environment, and other parasitic processes occurring in the set-up (such as Raman emission from optical fibers) hide the signal given by time-correlated photons. This is due to the severe attenuation of the processes. Nevertheless, we can estimate a lower bound for the SNR by analysing the coincidences between $\mathrm{D}_{1}$ and $\mathrm{D}_{4}$. We integrate the peak due to d-SFWM over its FWHM (16 bins), and we divide it by the noise, which is calculated as the average on the black histogram in Fig.5 multiplied by 16 bins. By taking into account the losses, we obtain a SNR equal to $(11.3 \pm 3.2) \cdot 10^{3}$. From the model, we should expect that the SNR is around $21 \cdot 10^{3}$, an improvement over that of a single ring system by over four orders of magnitude. Our experimental estimation is lower, since we are overestimating the noise due to s-SFWM.

These results demonstrate that the use of linearly un-

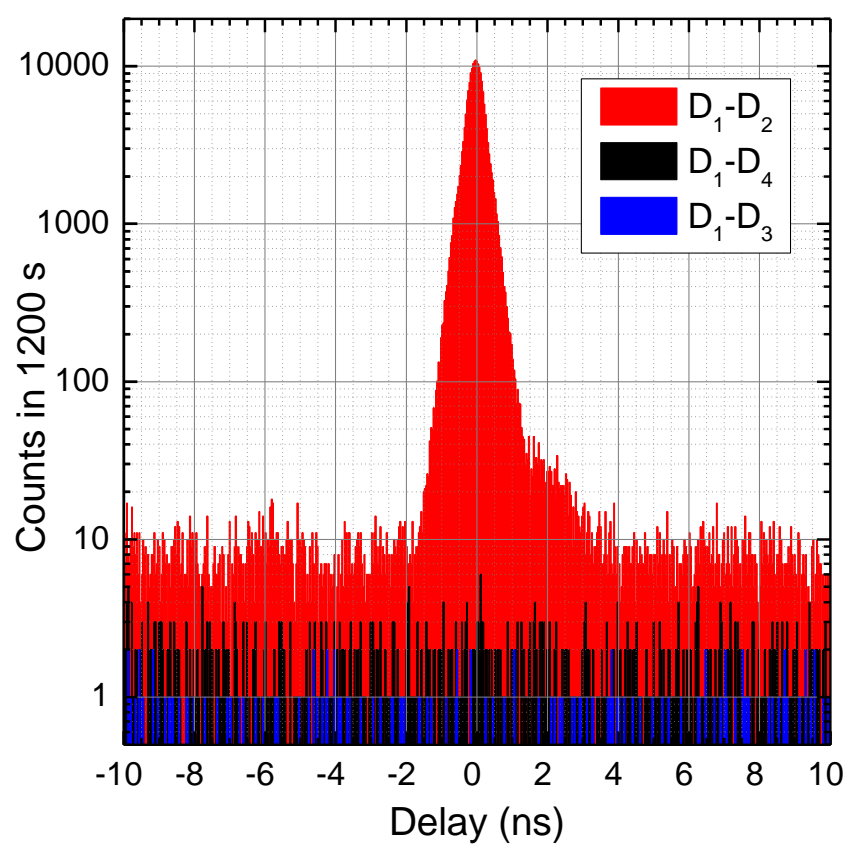

FIG. 5. Histograms representing the coincidences events detected. The red, black, and blue histograms represent, respectively, the coincidences due to d-SFWM (events on $\mathrm{D}_{1}$ and $\mathrm{D}_{2}$ ), $\mathrm{P}_{2}$-SFWM (events on $\mathrm{D}_{1}$ and $\mathrm{D}_{4}$ ), and $\mathrm{P}_{1}$-SFWM (events on $\mathrm{D}_{1}$ and $\mathrm{D}_{3}$ ). The width of each bin is $35 \mathrm{ps}$. The delay is calculated by taking into account the different optical lengths of the fibers from the device to the detectors. coupled resonators allows for the effective control of the nonlinear interaction between optical modes, leading to the ability to enhance or suppress the generation of photon pairs through spontaneous four wave mixing. This kind of device will be a central component in strategies for the implementation of continuous-variable quantum computing. It will enable the possibility of realising sources of highly squeezed light, since the main sources of noise, due to single-pump spontaneous four wave mixing, can be effectively suppressed. In our experiment, we could confirm the improvement of the signal-to-noise ratio by over four orders of magnitude.

This work has been supported by Ministero dell'Istruzione, dell'Università e della Ricerca (Dipartimenti di Eccellenza Program (2018-2022)). J.E.S. acknowledges support from the Natural Sciences and Engineering Research Council of Canada. The device has been designed using the open source Nazca $\operatorname{design}^{\mathrm{TM}}$ framework.

\section{REFERENCES}

[1] P. P. Absil, J. V. Hryniewicz, B. E. Little, P. S. Cho, R. A. Wilson, L. G. Joneckis, and P.-T. Ho, Optics Letters 25, 554 (2000).

[2] D. Grassani, S. Azzini, M. Liscidini, M. Galli, M. J. Strain, M. Sorel, J. E. Sipe, and D. Bajoni, Optica 2, 88 (2014).

[3] N. C. Harris, D. Grassani, A. Simbula, M. Pant, M. Galli, T. Baehr-Jones, M. Hochberg, D. Englund, D. Bajoni, and C. Galland, Physical Review X 4, 1 (2014).

[4] J. W. Silverstone, R. Santagati, D. Bonneau, M. J. Strain, M. Sorel, J. L. O'Brien, and M. G. Thompson, Nature Communications 6 (2015).

[5] M. Kues, C. Reimer, P. Roztocki, L. R. Cortés, S. Sciara, B. Wetzel, Y. Zhang, A. Cino, S. T. Chu, B. E. Little, D. J. Moss, L. Caspani, J. Azaña, and R. Morandotti, Nature 546, 622 (2017).

[6] P. Imany, J. A. Jaramillo-Villegas, O. D. Odele, K. Han, D. E. Leaird, J. M. Lukens, P. Lougovski, M. Qi, and A. M. Weiner, Optics Express 26, 1825 (2018).

[7] X. Lu, S. Rogers, T. Gerrits, W. C. Jiang, S. W. Nam, and Q. Lin, Optica 3, 1331 (2016).

[8] Y. Liu, C. Wu, X. Gu, Y. Kong, X. Yu, R. Ge, X. Cai, X. Qiang, J. Wu, X. Yang, and P. Xu, Optics Letters 45, 73 (2020).

[9] Y. Zhang, M. Menotti, K. Tan, V. D. Vaidya, D. H. Mahler, L. Zatti, M. Liscidini, B. Morrison, and Z. Vernon, (2020), arXiv:2001.09474.

[10] Y. Zhao, Y. Okawachi, J. K. Jang, X. Ji, M. Lipson, and A. L. Gaeta, Physical Review Letters 124, 193601 (2020).

[11] Z. Vernon, N. Quesada, M. Liscidini, B. Morrison, M. Menotti, K. Tan, and J. E. Sipe, Physical Review Applied 12, 1 (2019).

[12] Y. Guo, W. Zhang, S. Dong, Y. Huang, and J. Peng, Optics InfoBase Conference Papers 39, 2526 (2014).

[13] L. G. Helt, M. Liscidini, and J. E. Sipe, Journal of the Optical Society of America B 29, 2199 (2012). 
[14] C. M. Gentry, X. Zeng, and M. A. Popović, Optics Letters 39, 5689 (2014).

[15] M. Heuck, J. G. Koefoed, J. B. Christensen, Y. Ding, L. H. Frandsen, K. Rottwitt, and L. K. Oxenlowe, New Journal of Physics 21 (2019).

[16] M. Menotti, B. Morrison, K. Tan, Z. Vernon, J. E. Sipe, and M. Liscidini, Physical Review Letters 122, 13904 (2019).

[17] C. Bellegarde, E. Pargon, C. Sciancalepore, C. PetitEtienne, V. Hugues, D. Robin-Brosse, J.-M. Hartmann, and P. Lyan, IEEE Photonics Technology Letters 30, 591 (2018).
[18] F. A. Sabattoli, H. El Dirani, F. Garrisi, S. Sam, C. PetitEtienne, J. M. Hartmann, E. Pargon, C. Monat, M. Liscidini, C. Sciancalepore, M. Galli, and D. Bajoni, in 2019 21st International Conference on Transparent Optical Networks (ICTON) (2019) pp. 1-4.

[19] H. El Dirani, L. Youssef, C. Petit-Etienne, S. Kerdiles, P. Grosse, C. Monat, E. Pargon, and C. Sciancalepore, Optics Express 27, 30726 (2019).

[20] M. H. P. Pfeiffer, J. Liu, A. S. Raja, T. Morais, B. Ghadiani, and T. J. Kippenberg, Optica 5, 884 (2018).

[21] Q. Lin, O. J. Painter, and G. P. Agrawal, Optics Express 15, 16604 (2007). 